\title{
Improving Blanking/Clipping Based Impulsive Noise Mitigation over Powerline Channels
}

\author{
Khaled M. Rabie and Emad Alsusa \\ Microwave and Communication Systems (MACS) Group, \\ School of Electrical and Electronic Engineering, \\ The University of Manchester, United Kingdom, M13 9PL, \\ Email: \{khaled.rabie, e.alsusa\}@manchester.ac.uk
}

\begin{abstract}
Powerline communication technology is a promising communication platform for smart grid and has nowadays become an attractive alternative for data transmission in the home. Iimpulsive noise (IN) over such channels, however, remains the main factor responsible for degrading communication signals. Many techniques for mitigating IN have been reported in the literature the most common of which is preceding the OFDM receiver with blanking, clipping or hybrid (combined blanking-clipping) nonlinear preprocessors. In this paper, we propose to enhance the capability of these techniques by preprocessing the signal at the transmitter. A closedform analytical expression for the probability of IN detection error is derived and the problem of blanking/clipping threshold selection is also considered. The results reveal that the proposed is able to minimize the probability of IN detection error significantly and can provide up to $3 \mathrm{~dB}$ SNR improvement relative to the conventional techniques.
\end{abstract}

Index Terms-Blanking, clipping, impulsive noise, peak to average power ratio (PAPR), powerline communications (PLC), smart grid.

\section{INTRODUCTION}

$\mathbf{P}$ OWERLINE communication (PLC) is considered the backbone of smart grid and becomes even more appealing in harsh wireless environments where propagation loss is high such as in underground structures and buildings with metal walls. Since powerlines have not been designed for communication signals, in order to improve the reliability of PLC, it is essential to overcome a number of challenges such as the varying impedance of the wiring, high levels of frequency-dependent attenuation and the noise. Noise over powerlines is divided into two categories colored background noise and impulsive noise (IN) [1], [2] with the latter being the most dominant factor degrading the PLC signals. In this paper we adopt the well-know IN model, Middleton class-A model, [1], [3], which has been widely accepted in the literature to analyze and evaluate system perfoamance over PLC channels.

Several methods have been reported in the literature to improve the performance of OFDM based receivers in the presence of IN. One of the simplest and most efficient of which is to precede the conventional OFDM demodulator with a nonlinear preprocessor such as blanking, clipping or hybrid (combined blanking/clipping) [4]. Theoretical performance analysis to find closed-form expressions for the signal-to-noise ratio (SNR) at the output of the nonlinear preprocessors and optimization of the threshold is considered in [5]. In this paper we refer to this method as the unmodified method. Imperfect recognition of the IN signal may lead to nulling/clipping uncorrupted signal samples leading to IN detection errors and hence performance deterioration.
To the best of our knowledge, all studies on the topic of mitigating IN are based on entirely countering IN at the receiver side. Unlike these studies, in this paper, it is proposed that the OFDM signal is preprocessed at the transmitter in such a way to minimize the probability of IN detection error at the receiver. This could be done simply by applying a peak to average power ratio (PAPR) reduction technique. In this paper, we exploit the selective mapping (SLM) scheme [6] as it is well known for its robustness, and combine it with blanking, clipping or hybrid at the receiver to reduce IN. The contribution of this paper is twofold. First we derive a closed-form expression for the probability of IN detection error and, for more quantitative characterization, the corresponding output SNR is also considered. The second contribution resides in addressing the problem of threshold optimization under various PAPR scenarios for the three nonlinear preprocessors. The results reveal that minimizing the PAPR can also minimize the probability of IN detection error significantly and provide up to $3 \mathrm{~dB}$ SNR enhancement relative to the unmodified method.

The rest of the paper is organized as follows. In Section II, the system model is presented. The proposed technique is described in Section III. In Section IV, a theoretical expression for the probability of IN detection error is derived. Section V outlines the simulation results including output SNR performance and blanking/clipping threshold optimization. Finally conclusions are drawn in Section VI.

\section{System Model Overview}

In this section the system model used in this study is discribed and is illustrated Fig. 1. First the information bits are mapped into 16QAM symbol which are then passed through an OFDM modulator to produce a time domain signal, $s(t)$, defined as

$$
s(t)=\frac{1}{\sqrt{N}} \sum_{k=0}^{N-1} S_{k} e^{\frac{j 2 \pi k t}{T_{s}}}, \quad 0<t<T_{s}
$$

where $S_{k}$ is the complex constellations of the data symbols, $N$ is number of sub-carriers and $T_{s}$ is the active symbol interval. The PAPR of the transmitted signal is given by

$$
\operatorname{PAPR}=\frac{\max |s(t)|^{2}}{\mathbb{E}\left[|s(t)|^{2}\right]}
$$

where $\mathbb{E}[$.$] is the expectation function. In this paper we consider$ a special case of Middleton class-A noise model in which IN is 


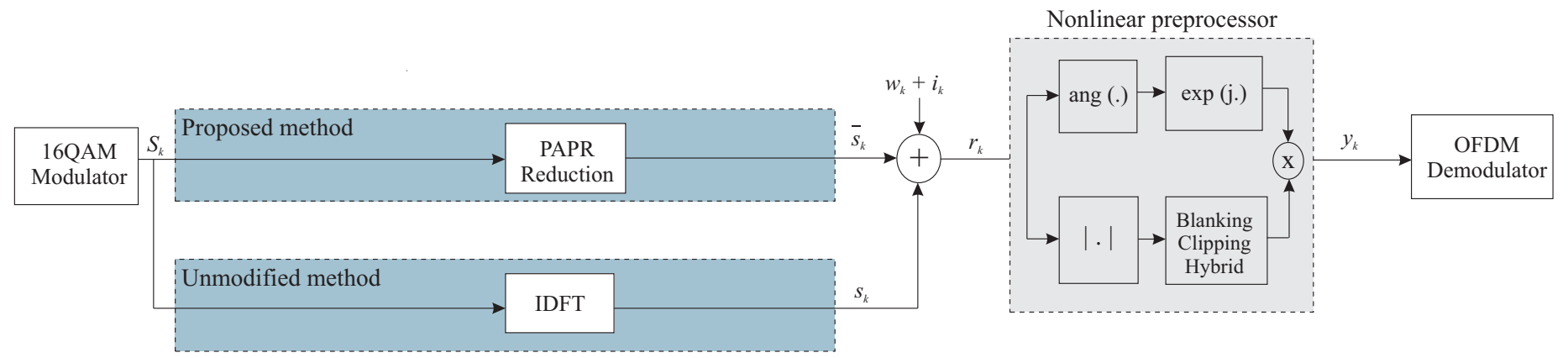

Fig. 1: Block diagram of the unmodified and proposed OFDM systems with nonlinear preprocessors at the receiver

modeled as a Bernoulli-Gaussian random process [7] and is given as

$$
n_{k}=w_{k}+i_{k}, \quad k=0,1,2, \ldots, N-1
$$

where

$$
i_{k}=b_{k} g_{k}, \quad k=0,1,2, \ldots, N-1
$$

$n_{k}$ is the total noise component, $w_{k}$ is the additive white Gaussian noise (AWGN), $i_{k}$ is the IN, $b_{k}$ is the Bernoulli process with probability $P\left(b_{k}=1\right)=p$ and $g_{k}$ is complex white Gaussian noise with mean zero. The probability density function (PDF) of the total noise can be expressed as

$$
P_{n_{k}}\left(n_{k}\right)=(1-p) \mathcal{G}\left(n_{k}, 0, \sigma_{w}^{2}\right)+p \mathcal{G}\left(n_{k}, 0, \sigma_{w}^{2}+\sigma_{i}^{2}\right)
$$

$\mathcal{G}($.$) is the Gaussian PDF. \sigma_{w}^{2}$ and $\sigma_{i}^{2}$ are the AWGN and IN variances, respectively, and define the input SNR and signalto-impulsive noise ratio (SINR) as $\mathrm{SNR}=10 \log _{10}\left(1 / \sigma_{w}^{2}\right)$, SINR $=10 \log _{10}\left(1 / \sigma_{i}^{2}\right)$. Under perfect synchronization condition, the received signal has the following form

$$
r_{k}=\left\{\begin{array}{ll}
s_{k}+w_{k}, & \mathcal{H}_{0} \\
s_{k}+w_{k}+i_{k}, & \mathcal{H}_{1}
\end{array} \quad k=0,1, \ldots, N-1\right.
$$

where $s_{k}=s\left(k T_{s} / N\right) ; s_{k}, w_{k}$ and $i_{k}$ are assumed to be mutually independent. The null hypothesis $\mathcal{H}_{0}$ implies the absence of IN, $P\left(\mathcal{H}_{0}\right)=(1-p)$, whereas the alternative hypothesis $\mathcal{H}_{1}$ implies the presence of IN, $P\left(\mathcal{H}_{1}\right)=p$. At the receiver, before the OFDM demodulator, the received signal is fed into the nonlinear preprocessor as shown in Fig. 1. The output of these devices are

- Blanking

$$
y_{k}=\left\{\begin{array}{ll}
r_{k}, & \left|r_{k}\right| \leq T_{b} \\
0, & \left|r_{k}\right|>T_{b}
\end{array} \quad k=0,1, \ldots, N-1\right.
$$

where $T_{b}$ is the blanking threshold.

- Clipping

$$
y_{k}=\left\{\begin{array}{ll}
r_{k}, & \left|r_{k}\right| \leq T_{c} \\
T_{c} e^{j a r g(r k)}, & \left|r_{k}\right|>T_{c}
\end{array} \quad k=0,1, \ldots, N-1\right.
$$

where $T_{c}$ is the clipping threshold.

- Hybrid

$$
y_{k}= \begin{cases}r_{k}, & \left|r_{k}\right| \leq T_{c} \\ T_{c} e^{j a r g(r k)}, & T_{c}<\left|r_{k}\right| \leq T_{b} \quad k=0,1, \ldots, N-1 \\ 0, & \left|r_{k}\right|>T_{b}\end{cases}
$$

where $r_{k}$ and $y_{k}$ are the input and output of the nonlinear preprocessors, respectively. The selection of the threshold value(s) is the key to maximize the system performance. On one hand, for very small threshold(s), most of the received samples will be blanked/clipped resulting in poor performance. On the other hand, if the threshold is too large, IN will be overlooked and will become part of the detected signal hence dramatically degrading performance. In [5], theoretical expressions for the optimal blanking, clipping and hybrid thresholds of the unmodified method were derived as a function of IN parameters as well as the output SNR. These expressions will be used to provide a comparative analysis to show the superiority of our proposed technique.

\section{The PRoposed METHOD}

As stated earlier, in this paper we propose preprocessing the OFDM signal at the transmitter to improve the noise cancellation process at the receiver. It is intuitive to think that if the average PAPR of the OFDM symbols is small, then this will make IN more distinguishable from the useful transmitted signal and therefore can be blanked/clipped more effectively at the receiver. This can be accomplished simply by deploying a well-known PAPR reduction method such as the SLM scheme. For further clarity, an illustrative example is presented in Fig. 2 showing plots of an unmodified OFDM signal, an SLM-OFDM signal and IN pulses. This presents two different scenarios. First, in the case of the unmodified system it can be seen that when the threshold $T_{1}$ is considered, two IN pulses will be recognized $\{\mathrm{IN} 2$, IN 3$\}$ whereas IN1 remains undetected which then becomes part of the signal fed to the OFDM demodulator. Whereas if $T_{2}$ is used, the nonlinear preprocessor will be able to identify $\{$ IN1, IN2, IN3\}; however, the unaffected samples $\{\mathrm{S} 1, \mathrm{~S} 2, \mathrm{~S} 3\}$ will also trigger the nonlinear device and consequently will be blanked/clipped causing an IN detection error. On the other hand, the SLM-OFDM system allows using $T_{2}$ without any blanking/clipping errors (leaving the unaffected samples untouched) in addition to eliminating (IN1, IN2, IN3 . The amount of reduction in blanking/clipping threshold is referred to as threshold gain ( $\mathrm{TG}=T_{2}-T_{1}$ ). It will be shown later that the higher the TG, the more performance enhancement is achieved in term of the output SNR. For better realization of the proposed technique, it is important to briefly review the operation of the SLM scheme.

In SLM scheme, the transmitter generates a set of different data blocks representing the same information as the original data block and then selects the one with the minimum PAPR for transmission. Assuming that the data stream is defined as $S=\left[S_{0}, S_{1}, \ldots, S_{N-1}\right]^{T}$, then each data block $S$ is multiplied 


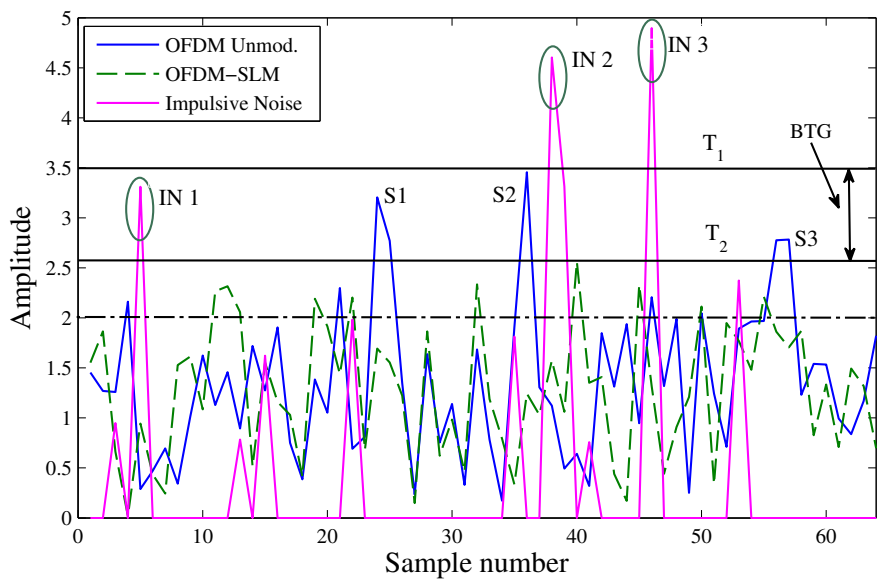

Fig. 2: Improved blanking/clipping threshold for 16QAM-SLM-OFDM system with $N=64$

by $U$ different phase sequence vectors $W$ of length $N$

$$
W^{(u)}=\left[W_{0}^{(u)}, W_{1}^{(u)}, \ldots, W_{N-1}^{(u)}\right]^{T} u=1,2, \ldots, U
$$

This multiplication yields $U$ modified data blocks

$$
\bar{S}^{(u)}=\left[S_{0}^{(u)} W_{0}^{(u)}, W_{1}^{(u)} W_{1}^{(u)}, \ldots, S_{N-1}^{(u)} W_{N-1}^{(u)}\right]^{T}
$$

The modified blocks are then passed through the IDFT and the SLM-OFDM signal with $N$ sub-carriers is given as

$$
s^{(u)}(t)=\frac{1}{\sqrt{N}} \sum_{k=0}^{N-1} \bar{S}_{k}^{(u)} e^{\frac{j 2 \pi k t}{T_{s}}}, \quad 0<t<T_{s}
$$

The modified data block with the minimum PAPR is selected for transmission, $\bar{s}(t)$. The amount of PAPR reduction improves as $U$ increases [6]. This reduction implies that more of the transmitted signal energy is contained close to the average value and hence larger TG value can be obtained.

\section{The Probability of IN Detection Error Analysis}

The probability of IN detection error $\left(P_{d_{e}}\right)$ is the probability that the amplitude of the received sample, $A_{r}=\left|r_{k}\right|$, exceeds the blanking/clipping threshold when it is unaffected by IN and it is expressed as

$$
\begin{gathered}
P_{d_{e}}=P\left(D \mid \mathcal{H}_{0}\right)=P\left(A_{r}>T \mid \mathcal{H}_{0}\right) P\left(\mathcal{H}_{0}\right) \\
=\left[1-F_{A_{r}}\left(T \mid \mathcal{H}_{0}\right)\right] P\left(\mathcal{H}_{0}\right)
\end{gathered}
$$

where $F_{A_{r}}\left(T \mid \mathcal{H}_{0}\right)$ is the conditional cumulative distribution function (CDF) and $D$ is the event of blanking/clipping the received signal exceeding $T$. In the absence of IN, the amplitude of the received signal has Rayleigh distribution with parameter $\sigma^{2}=\sigma_{s}^{2}+\sigma_{w}^{2}$. Therefore, for the unmodified method $P_{d_{e}}$ is

$$
P_{d_{e}}^{(\text {unmod })}=e^{-\frac{T^{2}}{2\left(\sigma_{s}^{2}+\sigma_{w}^{2}\right)}}(1-p)
$$

In the case of the SLM-OFDM system, the PDF of the transmitted signal as a function of $N$ and $U$ is derived in [8] and reproduced for convenience in (15). The conditional CDF of the SLM-OFDM system in the absence of IN is

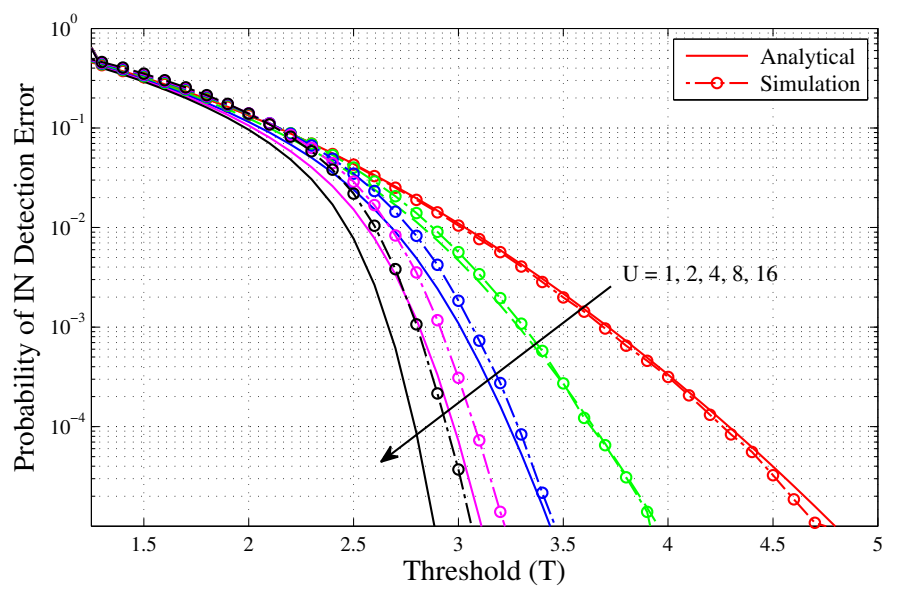

Fig. 3: Probability of IN detection error for both unmodified and proposed techniques with various values of $U$ and $N=64$

$$
\begin{gathered}
F_{A_{r}}^{S L M}\left(T \mid \mathcal{H}_{0}\right)=\left.\int_{-\infty}^{T} f^{S L M}(x) \mathrm{d} x\right|_{\sigma^{2}=\sigma_{s}^{2}+\sigma_{w}^{2}} \\
=\left(1-\left[1-\left(1-e^{-\frac{T^{2}}{2\left(\sigma_{s}^{2}+\sigma_{w}^{2}\right)}}\right)^{N}\right]^{U}\right)^{\frac{1}{N}}
\end{gathered}
$$

Using the definition of $P_{d_{e}}$ in (13) we can write the probability of IN detection error for the SLM-OFDM system $\left(P_{d_{e}}^{(\text {Proposed })}\right)$ as in (18). Some numerical results obtained from (18) are shown in Fig. 3 along with simulation results for an OFDM system with $\{N=64\}$ and input SNR $=40 \mathrm{~dB}$ for various values of $U$. It is clear that the simulation results closely match the analytical ones. From this figure, it is obvious that the behavior of the probability of IN detection error can be divided into two regions. The first region is when $\{T \lesssim 2\}$ during which the proposed system does not provide any probability reduction. It is clear that when $\{T=2\}$, about $\{\simeq 10 \%\}$ of the signal samples will exceed this threshold regardless of the number of phase sequences being used. This can also be clearly observed from Fig. 2 where about 7 samples out of 64 for each system exceed 2 (dashed line), which represents about $10 \%$ of the total samples. In the second region $\{T>2\}$ it is noticeable that the proposed minimizes the probability of IN detection error and that the probability is inversely proportional to $U$ and $T$. For instance when $\{U=16\}$ and at $T=2.5$, the probability is reduced by about 0.5 order of magnitude whereas for $T=3$, the probability is minimized by about 3 orders of magnitude. This implies that the system performance will improve for higher values of $U$ as will be further discussed in the next section.

\section{Simulation Results}

This section presents the performance of the proposed technique in terms of the SNR at the output of the blanking, clipping and hybrid devices in addition to the optimal blanking, clipping and hybrid thresholds that maximize the output SNR. It should be noted that the analytical results of the unmodified method $(U=1)$ are obtained using the expressions in [5]. Our simulation parameters are: $N=64, \sigma_{s}^{2}=(1 / 2) \mathbb{E}\left[\left|s_{k}\right|^{2}\right]=1, \sigma_{w}^{2}=(1 / 2) \mathbb{E}\left[\left|w_{k}\right|^{2}\right]$, 


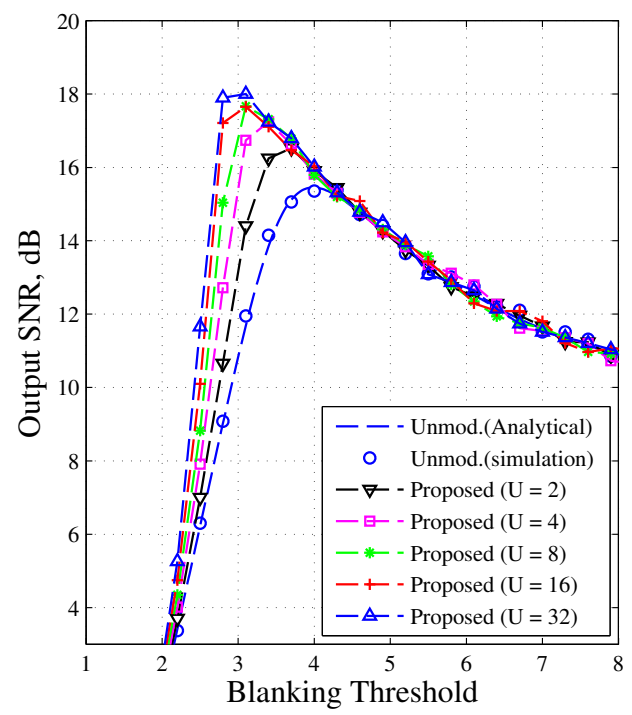

(a) Blanking

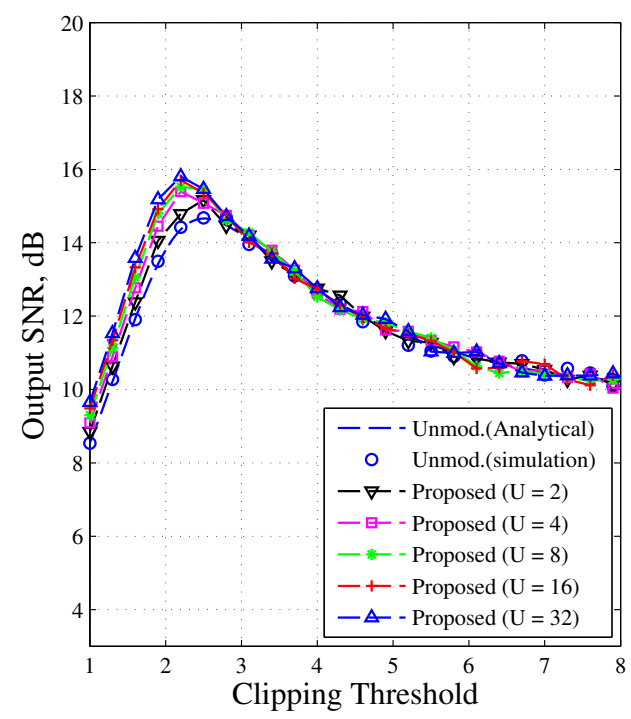

(b) Clipping

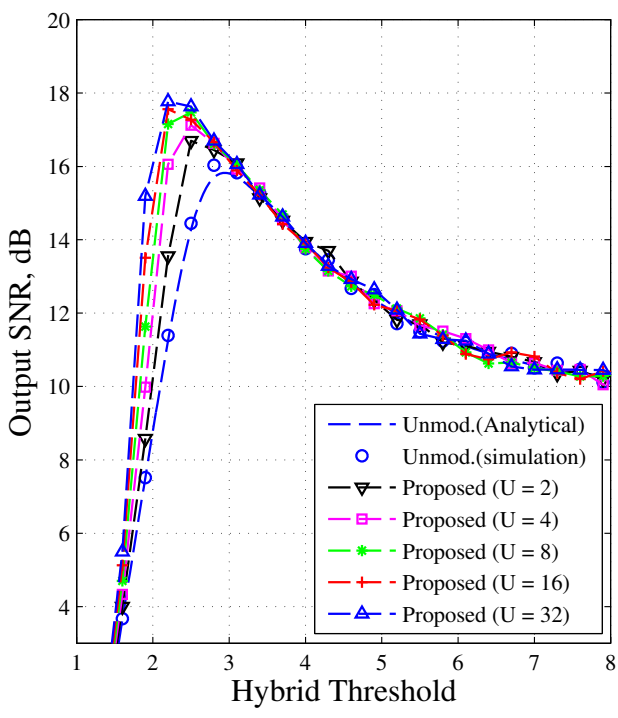

(c) Hybrid $T_{b}=1.4 T_{c}$

Fig. 4: The Output SNR versus blanking/clipping/hybrid threshold for different values of $U, p=0.01$, SNR $=40 \mathrm{~dB}$ and $\mathrm{SINR}=-10 \mathrm{~dB}$

$$
f^{S L M}(x)=U f(x)\left(\left(1-e^{-\frac{x^{2}}{2 \sigma^{2}}}\right)^{N}\right)^{U-1}\left(1-\left(1-\left(1-e^{-\frac{x^{2}}{2 \sigma^{2}}}\right)^{N}\right)^{U}\right)^{\frac{1}{N}}
$$

$$
P_{d_{e}}^{\text {proposed }}=\left(1-F_{A_{r}}^{S L M}\left(T \mid \mathcal{H}_{0}\right)\right) P\left(\mathcal{H}_{0}\right)=\left[1-\left(1-\left(1-\left(1-e^{\left(-\frac{T^{2}}{2\left(\sigma_{s}^{2}+\sigma_{w}^{2}\right)}\right)}\right)^{N}\right)^{U}\right)^{\frac{1}{N}}\right](1-p)
$$

$\sigma_{i}^{2}=(1 / 2) \mathbb{E}\left[\left|i_{k}\right|^{2}\right]$, SNR $=40 \mathrm{~dB}$ and $p=0.01$. The output SNR is found by (19) with $\overline{s_{k}}=\bar{s}\left(k T_{s} / N\right)$.

$$
\mathrm{SNR}_{\text {Proposed }}^{U}=\frac{\mathbb{E}\left[\left|\overline{s_{k}}\right|^{2}\right]}{\mathbb{E}\left[\left|y_{k}-\overline{s_{k}}\right|^{2}\right]}
$$

\section{A. The Output SNR versus Blanking/Clipping Threshold}

The SNR at the output of the blanking, clipping and hybrid preprocessors versus the threshold values is shown in Fig. 4a, 4b and $4 \mathrm{c}$, respectively, for $U=\{1,2,4,8,16,32\}$. It is noticeable that the proposed technique always performs better even for a small number phase sequences (e.g. $U=2$ ) and this enhancement is proportional to the value of $U$. It is also clear that when $T$ is too high $\{T \rightarrow \infty\}$, no blanking/clipping takes place and this allows all the IN energy to be part of the detected signal. In such scenario, it is obvious that the output SNR approaches $10 \mathrm{~dB}$ and this can be mathematically expressed as

$$
\operatorname{SNR}_{\text {Proposed }}^{U}(T \rightarrow \infty)=10 \log _{10}\left(\frac{\sigma_{s}^{2}}{\sigma_{w}^{2}+p \sigma_{i}^{2}}\right)
$$

When $p \sigma_{i}^{2} \gg \sigma_{w}^{2}$, (20) can be approximated to $\simeq$ $10 \log _{10}\left(1 /\left(p \sigma_{i}^{2}\right)\right)$. Furthermore, it can be noticed that for each value of $U$ there exists an optimal blanking, clipping or hybrid threshold which decreases as $U$ increases. The threshold optimization of the proposed system is investigated next.

\section{B. Threshold Optimization and Maximum Achievable SNR}

In this subsection extensive simulations have been conducted to optimize the blanking/clipping/hybrid threshold as

$$
T_{\text {opt }}^{U}=\arg \underset{0 \leq T<\infty}{\max }\left\{\operatorname{SNR}_{\text {Proposed }}^{U}(T, p, \operatorname{SINR}, \mathrm{SNR})\right\}
$$

The optimal threshold for the blanking, clipping and hybrid devices versus SINR are presented in Fig. 5a, 5b and 5c, respectively. From these plots it is observed that as $U$ increases, the optimal thresholds become smaller. Another interesting observation one can see is in Fig. 5a, where the optimal blanking threshold levels off for sufficiently larges value of $U$. This implies that the optimal blanking threshold becomes independent of IN characteristics for $U \geq 16$. Furthermore, the maximum achievable SNR at the output of the nonlinear preprocessors corresponding to the optimal blanking/clipping/hybrid threshold versus SINR is illustrated in Fig. 6. We can see that for the three systems, the proposed technique always outperforms the unmodified method. It is also evident that for the blanking and hybrid systems the gain is about $3 \mathrm{~dB}$ and $2 \mathrm{~dB}$, respectively, in the intermediate SINR region $(-5 \mathrm{~dB} \rightarrow-15 \mathrm{~dB})$ when $U=32$. However, for the clipping scenario, the gain remains constant for almost all the SINR spectrum at about $1.25 \mathrm{~dB}$ for the same value of $U$. 


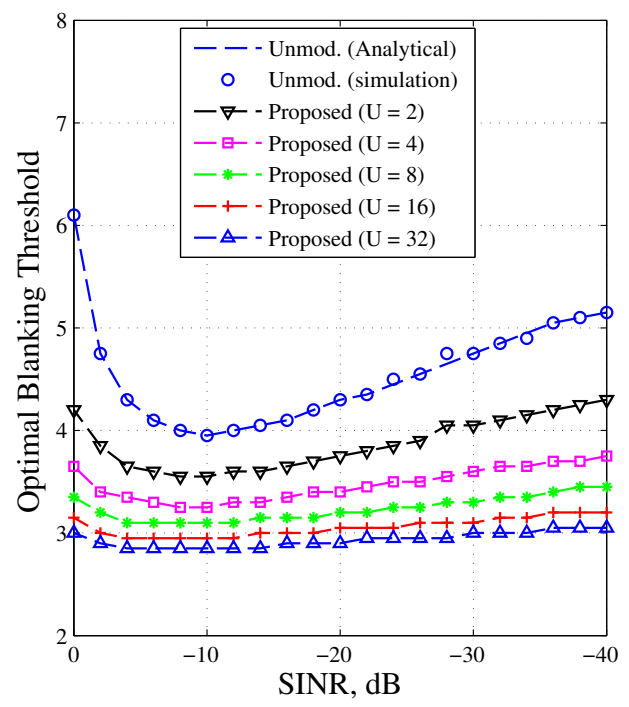

(a) Blanking

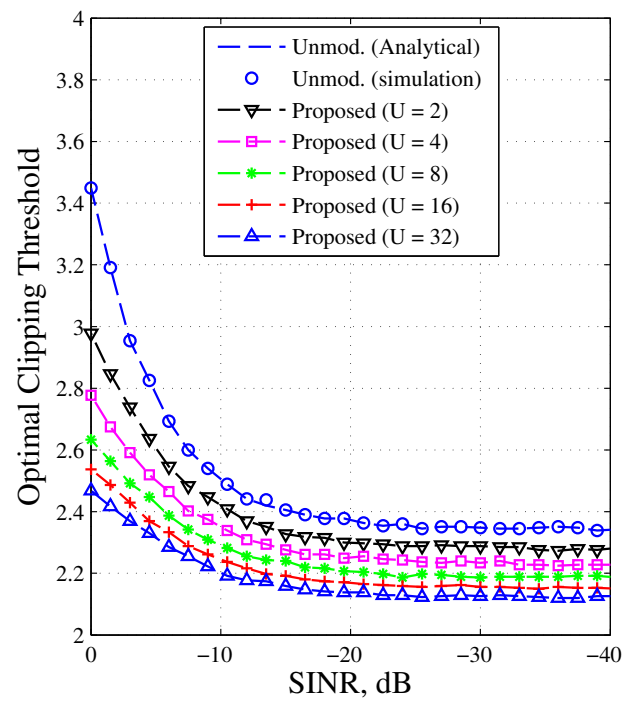

(b) Clipping

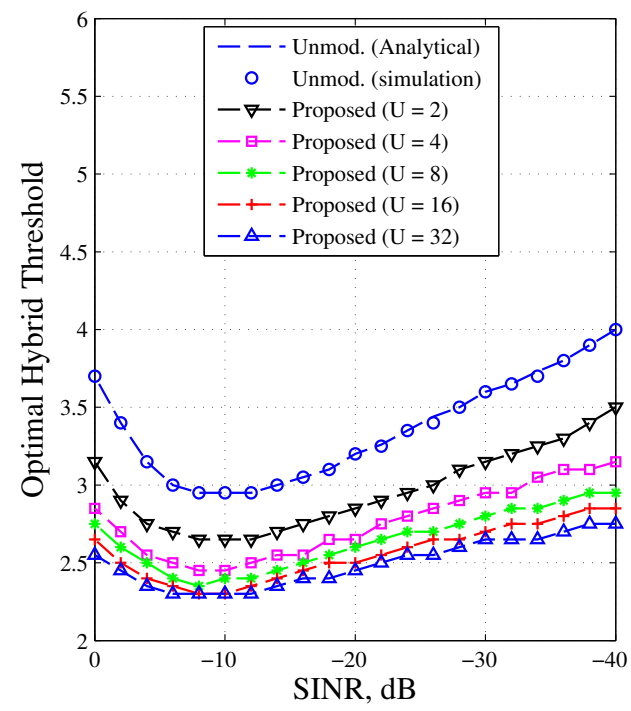

(c) Hybrid $T_{b}=1.4 T_{c}$

Fig. 5: Optimal blanking/clipping threshold versus SINR for different values of $U, p=0.01$ and $\mathrm{SNR}=40 \mathrm{~dB}$

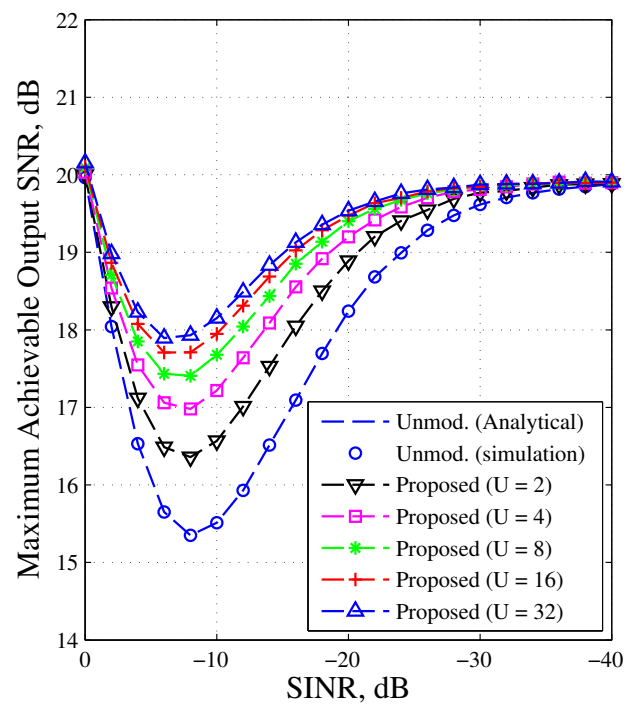

(a) Blanking

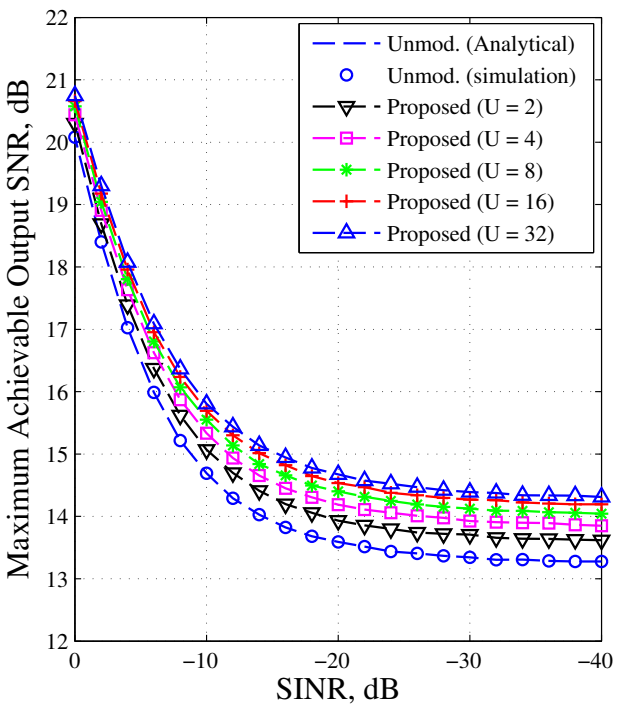

(b) Clipping

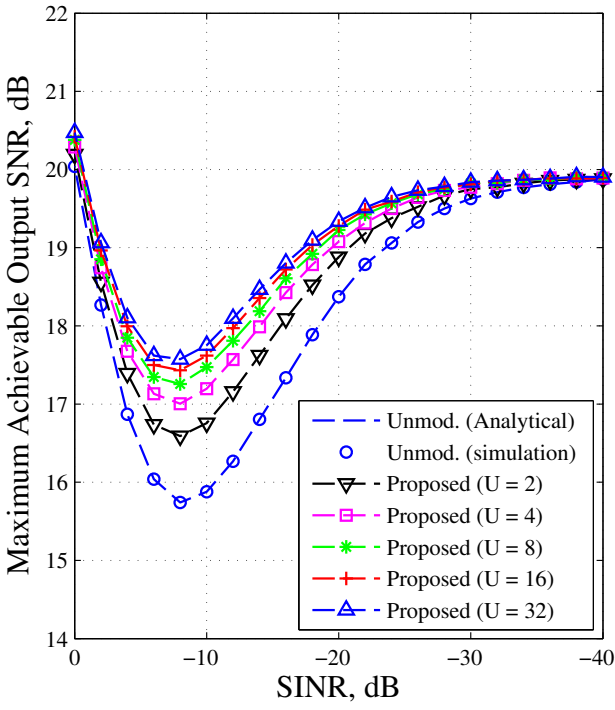

(c) Hybrid $T_{b}=1.4 T_{c}$

Fig. 6: The maximum achievable output SNR as a function of SINR for various values of $U, p=0.01$ and SNR $=40 \mathrm{~dB}$

\section{CONCLUSION}

In this paper we introduced a technique to improve the conventional OFDM receivers with nonlinear preprocessors in the presence of IN by utilizing a PAPR reduction technique. The problem of threshold optimization is also investigated and the corresponding maximum achievable output SNR is demonstrated. The results reveal that the proposed can considerably reduce the probability of IN detection error which consequently maximizes the output SNR. Furthermore, it was found that when $U=32$, the suggested scheme can attain gains up to $3 \mathrm{~dB}, 1.25 \mathrm{~dB}$ and $2 \mathrm{~dB}$ for blanking, clipping and hybrid preprocessors, respectively, in the intermediate SINR region.

\section{REFERENCES}

[1] D. Middleton, "Canonical and quasi-canonical probability models of class-A interference," IEEE Trans. Electromagn. Compat., vol. EMC-25, pp. 76-106,
May 1983.

[2] - "Non-gaussian noise models in signal processing for telecommunications: new methods an results for class A and class B noise models," IEEE Trans. Inform. Theory, vol. 45, no. 4, pp. 1129 -1149, May 1999.

[3] — "Statistical-physical models of electromagnetic interference," IEEE Trans. Electromagn. Compat., vol. EMC-19, pp. 106-127, Aug. 1977.

[4] O. P. H. et al., "Detection and removal of clipping in multicarrier receivers," European patent application EP1043874, Oct. 2011.

[5] S. V. Zhidkov, "Analysis and comparison of several simple impulsive noise mitigation schemes for OFDM receivers," IEEE Trans. Commun., vol. 56, no. 1, pp. 5-9, Jan. 2008.

[6] R. W. Baaauml, R. F. H. Fisher, and J. B. Huber, "Reducing the peak-toaverage power ratio of multicarrier modulation by selected mapping," Elect. Lett., vol. 32, no. 22, pp. 2056-57, Oct. 1996.

[7] M. Ghosh, "Analysis of the effect of impulse noise on multicarrier and single carrier QAM systems," IEEE Trans. Commun., vol. 44, no. 2, pp. 145-147, Feb. 1996.

[8] H. Yoo, F. Guilloud, and R. Pyndiah, "Amplitude PDF analysis of OFDM signal using probabilistic PAPR reduction method," EURASIP Journal on Wireless Communications and Networking, vol. 2011, 2011. 